\title{
SOLVING VARIATIONAL INCLUSIONS BY A MULTIPOINT ITERATION METHOD UNDER CENTER-HÖLDER CONTINUITY CONDITIONS
}

Abstract. We prove the existence of a sequence $\left(x_{k}\right)$ satisfying $0 \in f\left(x_{k}\right)$ $+\sum_{i=1}^{M} a_{i} \nabla f\left(x_{k}+\beta_{i}\left(x_{k+1}-x_{k}\right)\right)\left(x_{k+1}-x_{k}\right)+F\left(x_{k+1}\right)$, where $f$ is a function whose second order Fréchet derivative $\nabla^{2} f$ satifies a center-Hölder condition and $F$ is a set-valued map from a Banach space $X$ to the subsets of a Banach space $Y$. We show that the convergence of this method is superquadratic.

1. Introduction. In a previous paper [5], we used a multipoint iteration formula to solve the "abstract" generalized equation

$$
0 \in f(x)+F(x)
$$

where $f$ is a function from $X$ into $Y$ which possesses a second order Fréchet derivative, $F$ is a set-valued map from $X$ to the subsets of $Y$ with closed graph, and $X, Y$ are two Banach spaces. We obtained cubic convergence when the second order Fréchet derivative is Lipschitz.

Recall that equation (1) is an abstract model for various problems:

- When $F=\{0\},(1)$ is an equation.

- When $F$ is the positive orthant in $\mathbb{R}^{m},(1)$ is a system of inequalities.

- When $F$ is the normal cone to a convex and closed set in $X$, (1) may represent variational inequalities.

Now, we recall some results obtained for the original problem. When the Fréchet derivative $\nabla f$ is locally Lipschitz, Dontchev $[6,7]$ associated to (1) a Newton-type method based on a partial linearization which provides local quadratic convergence. Following his work, Pietrus [19] obtained a

2000 Mathematics Subject Classification: 49J53, 47H04, 65K10.

Key words and phrases: set-valued mapping, generalized equations, Aubin continuity, pseudo-Lipschitz map, multipoint iteration formula, center-Hölder continuity. 
Newton-type sequence which converges whenever $\nabla f$ satisfies a Hölder-type condition, and in [20] he proved the stability.

Using a second degree Taylor polynomial expansion of $f$ at $x_{k}$, Geoffroy, Hilout and Pietrus [10] introduced a method involving the second order Fréchet derivative, and when $\nabla^{2} f$ is Lipschitz, they obtained cubic convergence. In [11], they proved the stability of the method. In [12], Geoffroy and Pietrus showed that the method is superquadratic when $\nabla^{2} f$ satisfies the Hölder condition. More recently, Jean-Alexis [15] presented a method without second order derivative, which is also cubically convergent, and Geoffroy, Jean-Alexis and Pietrus [13] showed the stability of the method when $\nabla^{2} f$ is Lipschitz. When $\nabla^{2} f$ is Hölder, Pietrus and Jean-Alexis [16] obtained superquadratic convergence. Our method generalizes this idea by taking more iterates.

To solve (1), for a fixed integer $M>1$ we consider a sequence $\left(x_{k}\right) \subset X$ satisfying

$$
0 \in f\left(x_{k}\right)+\sum_{i=1}^{M} a_{i} \nabla f\left(x_{k}+\beta_{i}\left(x_{k+1}-x_{k}\right)\right)\left(x_{k+1}-x_{k}\right)+F\left(x_{k+1}\right)
$$

where $\left(a_{i}\right)_{1 \leq i \leq M}$ and $\left(\beta_{i}\right)_{1 \leq i \leq M}$ are two sequences of real numbers satisfying

$$
\sum_{i=1}^{M} a_{i}=1 \quad \text { and } \quad \sum_{i=1}^{M} a_{i} \beta_{i}=\frac{1}{2} .
$$

This method was inspired by some multipoint iteration formula given in [23] for approximate $f$.

In [4], we showed the superquadratic convergence of the sequence defined by (2) when the second order Fréchet derivative of $f$ satisfies a Hölder condition.

Our aim in this work is to study the case where $\nabla^{2} f$ satisfies a Hölder condition centerd at a solution $x^{*}$ of (1). Such conditions have been introduced by Argyros [1] and are weaker than Hölder conditions.

The paper is organized as follows: in Section 2, we give some notations and definitions, and recall a few preliminary results including a fixed-point theorem (Lemma 2.1) which is the main tool for proving the existence and convergence of the sequence defined by (2) to a solution of equation (1); we also make some fundamental assumptions on $f$. Then, in Section 3, we prove the existence and convergence of the sequence in question. Moreover, we prove that the convergence of this method is superquadratic.

2. Notations, definitions and preliminary results. Let us recall some notations: 
- The distance from a point $x$ to a set $A$ in a metric space $(Z, \varrho)$ is defined by

$$
\operatorname{dist}(x, A)=\inf \{\varrho(x, y): y \in A\} .
$$

- The excess e from the set $A$ to the set $C$ is given by

$$
e(A, C)=\sup \{\operatorname{dist}(x, A): x \in C\} .
$$

- If $\Lambda: X \rightrightarrows Y$ is a set-valued map, we define

$$
\begin{aligned}
\operatorname{graph} \Lambda & =\{(x, y) \in X \times Y: y \in \Lambda(x)\}, \\
\Lambda^{-1}(y) & =\{x \in X: y \in \Lambda(x)\} .
\end{aligned}
$$

- $B_{r}(x)$ is the closed ball centerd at $x$ with radius $r$.

- The norms in the Banach spaces $X$ and $Y$ are both denoted by $\|\cdot\|$.

Now, we collect some results that we will need to prove our main theorem.

Definition 2.1. A set-valued $\Lambda$ is pseudo-Lipschitz around $\left(x_{0}, y_{0}\right) \in$ graph $\Lambda$ with modulus $M$ if there exist constants $a$ and $b$ such that

$$
\sup _{z \in \Lambda\left(y^{\prime}\right) \cap B_{a}\left(y_{0}\right)} \operatorname{dist}\left(z, \Lambda\left(y^{\prime \prime}\right)\right) \leq M\left\|y^{\prime}-y^{\prime \prime}\right\| \quad \text { for all } y^{\prime} \text { and } y^{\prime \prime} \text { in } B_{b}\left(x_{0}\right) .
$$

Using the excess, we have an equivalent definition replacing the inequality (4) by

$$
e\left(\Lambda\left(y^{\prime}\right) \cap B_{a}\left(y_{0}\right), \Lambda\left(y^{\prime \prime}\right)\right) \leq M\left\|y^{\prime}-y^{\prime \prime}\right\| \quad \text { for all } y^{\prime} \text { and } y^{\prime \prime} \text { in } B_{b}\left(x_{0}\right) .
$$

The pseudo-Lipschitz property has been introduced by J.-P. Aubin and he was the first to define this concept as a continuity property. Sometimes this property is called "Aubin continuity". Characterizations of the pseudoLipschitz property have also been obtained by Rockafellar [21, 22] using the Lipschitz continuity of the distance function $\operatorname{dist}(y, \Lambda(x))$ around $\left(x_{0}, y_{0}\right)$ and by Mordukhovich $[17,18]$ using the concept of coderivative of a multifunction. The Aubin continuity of $F$ is equivalent to the metric regularity of $F^{-1}$, so more recently, Dontchev, Quincampoix and Zlateva [9] gave a derivative criterion of metric regularity of set-valued mappings based on work of Aubin and co-authors. For more details and applications of this property, the reader is referred to $[3,2,8]$.

DeFinition 2.2. We say that a function $f$ from a metric space $(X, \varrho)$ into a metric space $(Y, d)$ is strictly stationary at $x_{0} \in X$ if, for every $\varepsilon>0$, there exists $\delta>0$ such that

$$
d\left(f\left(x_{1}\right), f\left(x_{2}\right)\right) \leq \varepsilon \varrho\left(x_{1}, x_{2}\right) \quad \text { whenever } \quad \varrho\left(x_{i}, x_{0}\right)<\delta, i=1,2 .
$$

Definition 2.3. We say that a function $f$ from $X$ into $Y$ admits a second order Fréchet derivative $\nabla^{2} f$ which satisfies the $\alpha$-center-Hölder 
condition at $x^{*}$ on an open set $\Omega \subset X$ with constant $K$ if

$$
\forall x \in \Omega, \quad\left\|\nabla^{2} f(x)-\nabla^{2} f\left(x^{*}\right)\right\| \leq K\left\|x-x^{*}\right\|^{\alpha} .
$$

We then also say that $\nabla^{2} f$ is $\alpha$-center-Hölder at $x^{*}$.

This concept is weaker than Hölder continuity. For more details on these two concepts, the reader is referred to [1].

Lemma 2.1. Let $(Z, \varrho)$ be a complete metric space, let $\phi$ be a set-valued map from $Z$ into the closed subsets of $Z$, let $\eta_{0} \in Z$ and let $r$ and $\lambda$ be such that $0 \leq \lambda<1$ and

(a) $\operatorname{dist}\left(\eta_{0}, \phi\left(\eta_{0}\right)\right) \leq r(1-\lambda)$,

(b) $e\left(\phi\left(x_{1}\right) \cap B_{r}\left(\eta_{0}\right), \phi\left(x_{2}\right)\right) \leq \lambda \varrho\left(x_{1}, x_{2}\right)$ for all $x_{1}, x_{2} \in B_{r}\left(\eta_{0}\right)$.

Then $\phi$ has a fixed point in $B_{r}\left(\eta_{0}\right)$, that is, there exists $x \in B_{r}\left(\eta_{0}\right)$ such that $x \in \phi(x)$. If $\phi$ is single-valued, then $x$ is the unique fixed point of $\phi$ in $B_{r}\left(\eta_{0}\right)$.

The proof of Lemma 2.1 is given in [8] employing the standard iteration method for contracting mappings. This fixed point lemma is a generalization of a fixed point theorem in Ioffe-Tikhomirov [14] where in (b), the excess $e$ is replaced by the Hausdorff distance.

In the remainder of this work, the distance $\varrho$ in Lemma 2.1 is replaced by the norm.

LEMMA 2.2. Let $f$ be a function whose second order Fréchet derivative is $\alpha$-center-Hölder at $x^{*}$ with constant $K$ in an open set $\Omega \subset X$. Then for all $x \in \Omega$,

$$
\begin{aligned}
\| f(x)-f\left(x^{*}\right)-\nabla f\left(x^{*}\right)\left(x-x^{*}\right)-\frac{1}{2} & \nabla^{2} f\left(x^{*}\right)\left(x-x^{*}\right)^{2} \| \\
& \leq \frac{K}{(\alpha+1)(\alpha+2)}\left\|x-x^{*}\right\|^{2+\alpha} .
\end{aligned}
$$

Lemma 2.2 is the same as in [5] with $x_{1}=x^{*}$ and the proof is given in [12]; the above inequality is widely used in the present work.

We make the following assumptions:

$(\mathcal{H} 1) \nabla^{2} f$ is $\alpha$-center-Hölder $\left.\left.(\alpha \in] 0,1\right]\right)$ with constant $K_{2}$ in a neighborhood $\Omega$ of $x^{*}$.

$(\mathcal{H} 2) \quad F$ is a set-valued map with closed graph.

$(\mathcal{H} 3)(f+F)^{-1}$ is pseudo-Lipschitz around $\left(0 ; x^{*}\right)$ with constants $a, b$ and modulus $L$.

Thanks to the assumption $(\mathcal{H} 1)$, we can suppose without loss of generality that $\nabla^{2} f$ is bounded on $\Omega$ by a constant $K_{1}$, which implies that $\nabla f$ is $K_{1}$-Lipschitz on $\Omega$. 
We also define the following functions:

$$
\begin{aligned}
\Lambda_{k}(x) & =f\left(x_{k}\right)+\sum_{i=1}^{M} a_{i} \nabla f\left(x_{k}+\beta_{i}\left(x-x_{k}\right)\right)\left(x-x_{k}\right), \\
\Lambda_{x^{*}}(x) & =f\left(x^{*}\right)+\sum_{i=1}^{M} a_{i} \nabla f\left(x^{*}+\beta_{i}\left(x-x^{*}\right)\right)\left(x-x^{*}\right), \\
Q(x) & =\Lambda_{x^{*}}(x)+F(x)
\end{aligned}
$$

and

$$
\Psi_{k}(x)=Q^{-1}\left(\Lambda_{x^{*}}(x)-\Lambda_{k}(x)\right)
$$

3. Description and convergence analysis. The outline of our method is as follows:

- From a starting point $x_{0}$ in a neighborhood of a solution $x^{*}$ of (1), applying Lemma 2.1, we show that $\Psi_{0}$ has a fixed point $x_{1}$.

- From a current iterate $x_{k}$ and a function $\Psi_{k}$ defined on $X$ by (7), applying Lemma 2.1, we obtain the existence of the next iterate $x_{k+1}$ which is a fixed point of $\Psi_{k}$.

The main result of this study is as follows:

TheOREM 3.1. Let $x^{*}$ be a solution of (1), $f$ a function whose second order Fréchet derivative satisfies $(\mathcal{H} 1)$, and $F: X \rightrightarrows Y$ a set-valued function which satisfies $(\mathcal{H} 2)-(\mathcal{H} 3)$. Then for all $c$ satisfying

$$
c>\frac{L K_{2}}{(1+\alpha)(2+\alpha)}\left(1+(2+\alpha) \sum_{i=1}^{M}\left|a_{i}\right|\left|1-\beta_{i}\right|^{1+\alpha}\right),
$$

one can find $\delta>0$ such that for every starting point $x_{0} \in B_{\delta}\left(x^{*}\right)$ with $x_{0} \neq x^{*}$, there exists a sequence $\left(x_{k}\right)_{k \geq 0}$ defined by (2) which satisfies

$$
\left\|x_{k+1}-x^{*}\right\| \leq c\left\|x_{k}-x^{*}\right\|^{2+\alpha} .
$$

To prove the theorem, we need the following result given in [5]:

Proposition 3.1. The following are equivalent:

(a) $\left[f\left(x^{*}\right)+\sum_{i=1}^{M} a_{i} \nabla f\left(x^{*}+\beta_{i}\left(\cdot-x^{*}\right)\right)\left(\cdot-x^{*}\right)+F(\cdot)\right]^{-1}$ is pseudoLipschitz around $\left(y^{*}, x^{*}\right)$.

(b) $(f+F)^{-1}$ is pseudo-Lipschitz around $\left(y^{*}, x^{*}\right)$.

Proposition 3.2. Under the assumptions of Theorem 3.1, there exists $\delta>0$ such that for all $x_{0} \in B_{\delta}\left(x^{*}\right)$ and $x_{0} \neq x^{*}$, the map $\Psi_{0}$ admits a fixed point $x_{1} \in B_{\delta}\left(x^{*}\right)$. 
Proof of Proposition 3.2. We prove both assumptions (a) and (b) of Lemma 2.1. The assumption $(\mathcal{H} 3)$ gives constants $a$ and $b$ and we set

$$
\begin{aligned}
\alpha_{1} & =\sqrt{\frac{2 b}{3 K_{1} \sum_{i=1}^{M}\left|a_{i}\right|\left(1+2\left|\beta_{i}\right|\right)}}, \\
\alpha_{2} & =\sqrt[1+\alpha]{1 / C} \\
\alpha_{3} & =\sqrt[2+\alpha]{\frac{b(1+\alpha)(2+\alpha)}{K_{2}\left(1+(2+\alpha) \sum_{i=1}^{M}\left|a_{i}\right|\left|1-\beta_{i}\right|^{1+\alpha}\right)}} .
\end{aligned}
$$

Fix $\delta>0$ such that

$$
\delta<\min \left\{a, \alpha_{1}, \alpha_{2}, \alpha_{3}\right\} .
$$

From the definition of the excess $e$, we have

$$
\operatorname{dist}\left(x^{*}, \Psi_{0}\left(x^{*}\right)\right) \leq e\left(Q^{-1}(0) \cap B_{\delta}\left(x^{*}\right), Q^{-1}\left\{\Lambda_{x^{*}}\left(x^{*}\right)-\Lambda_{0}\left(x^{*}\right)\right\}\right) .
$$

Set

We have

$$
y^{*}=\Lambda_{x^{*}}\left(x^{*}\right)-\Lambda_{0}\left(x^{*}\right) .
$$

so

$$
\left\|y^{*}\right\|=\left\|f\left(x^{*}\right)-f\left(x_{0}\right)-\sum_{i=1}^{M} a_{i} \nabla f\left(x_{0}+\beta_{i}\left(x^{*}-x_{0}\right)\right)\left(x^{*}-x_{0}\right)\right\|
$$

$$
\begin{aligned}
\left\|y^{*}\right\| \leq & \left\|f\left(x^{*}\right)-f\left(x_{0}\right)+\nabla f\left(x^{*}\right)\left(x_{0}-x^{*}\right)+\frac{1}{2} \nabla^{2} f\left(x^{*}\right)\left(x_{0}-x^{*}\right)^{2}\right\| \\
& +\|-\nabla f\left(x^{*}\right)\left(x_{0}-x^{*}\right)-\sum_{i=1}^{M} a_{i} \nabla f\left(x_{0}+\beta_{i}\left(x^{*}-x_{0}\right)\right)\left(x^{*}-x_{0}\right) \\
& -\frac{1}{2} \nabla^{2} f\left(x^{*}\right)\left(x_{0}-x^{*}\right)^{2} \|=: A+B .
\end{aligned}
$$

From Lemma 2.2, we obtain

$$
A \leq \frac{K_{2}}{(1+\alpha)(2+\alpha)}\left\|x_{0}-x^{*}\right\|^{2+\alpha} .
$$

On the other hand, by (3) we have

$$
B=\left\|\sum_{i=1}^{M} a_{i}\left(\nabla f\left(x^{*}\right)-\nabla f\left(x_{0}+\beta_{i}\left(x^{*}-x_{0}\right)\right)\right)\left(x^{*}-x_{0}\right)-\frac{1}{2} \nabla^{2} f\left(x^{*}\right)\left(x^{*}-x_{0}\right)^{2}\right\| \text {. }
$$

As

$$
\begin{aligned}
\sum_{i=1}^{M} a_{i}(\nabla & \left.f\left(x^{*}\right)-\nabla f\left(x_{0}+\beta_{i}\left(x^{*}-x_{0}\right)\right)\right)\left(x^{*}-x_{0}\right) \\
= & \sum_{i=1}^{M} a_{i}\left(1-\beta_{i}\right) \int_{0}^{1} \nabla^{2} f\left(x^{*}+t\left(x_{0}-x^{*}\right)+t \beta_{i}\left(x^{*}-x_{0}\right)\right) d t\left(x^{*}-x_{0}\right)^{2},
\end{aligned}
$$


with the use of $(\mathcal{H} 1)$ and $(3)$ we have

$$
\begin{aligned}
B \leq \sum_{i=1}^{M}\left|a_{i}\right|\left|1-\beta_{i}\right| \int_{0}^{1} \| \nabla^{2} f\left(x^{*}+t\left(x_{0}-x^{*}\right)+t \beta_{i}\left(x^{*}-x_{0}\right)\right) & -\nabla^{2} f\left(x^{*}\right) \| d t \\
\leq K_{2} \sum_{i=1}^{M}\left|a_{i}\right|\left|1-\beta_{i}\right|^{1+\alpha} \int_{0}^{1} t^{\alpha} d t \cdot\left\|x_{0}-x^{*}\right\|^{2 \alpha} & \times\left\|x_{0}-x^{*}\right\|^{2}
\end{aligned}
$$

and hence

$$
B \leq \frac{K_{2}}{1+\alpha} \sum_{i=1}^{M}\left|a_{i}\right|\left|1-\beta_{i}\right|^{1+\alpha}\left\|x_{0}-x^{*}\right\|^{2+\alpha} .
$$

According to (10) and (11), we have

$$
\left\|y^{*}\right\| \leq \frac{K_{2}}{(1+\alpha)(2+\alpha)}\left(1+(2+\alpha) \sum_{i=1}^{M}\left|a_{i}\right|\left|1-\beta_{i}\right|^{1+\alpha}\right)\left\|x_{0}-x^{*}\right\|^{2+\alpha}
$$

and using condition (9), we obtain

$$
\left\|y^{*}\right\| \leq b
$$

By hypothesis $(\mathcal{H} 3)$, since $Q^{-1}$ is $L$-pseudo-Lipschitz, we obtain

$$
\operatorname{dist}\left(x^{*}, \Psi_{0}\left(x^{*}\right)\right) \leq L\left\|\Lambda_{x^{*}}\left(x^{*}\right)-\Lambda_{0}\left(x^{*}\right)\right\|,
$$

which yields

$$
\begin{aligned}
& \operatorname{dist}\left(x^{*}, \Psi_{0}\left(x^{*}\right)\right) \\
& \leq \frac{L K_{2}}{(1+\alpha)(2+\alpha)}\left(1+(2+\alpha) \sum_{i=1}^{M}\left|a_{i}\right|\left|1-\beta_{i}\right|^{1+\alpha}\right)\left\|x^{*}-x_{0}\right\|^{2+\alpha} .
\end{aligned}
$$

By setting

$$
r=r_{0}=c\left\|x^{*}-x_{0}\right\|^{2+\alpha}, \quad \lambda=L K_{1} \delta\left[\sum_{i=1}^{M}\left|a_{i}\right|\left(1+4\left|\beta_{i}\right|\right)\right],
$$

where $c$ is as in Theorem 3.1, one can find $\delta$ small enough such that $\lambda \in] 0,1[$ and

$$
c(1-\lambda)>\frac{L K_{2}}{(1+\alpha)(2+\alpha)}\left(1+(2+\alpha) \sum_{i=1}^{M}\left|a_{i}\right|\left|1-\beta_{i}\right|^{1+\alpha}\right),
$$

and assumption (a) in Lemma 2.1 is satisfied. 
Note that the choice of $r_{0}$ implies $r_{0} \leq \delta<a$.

Now, let us show that assumption (b) is also satified. Let $x \in B_{\delta}\left(x^{*}\right)$ and set $z=\Lambda_{x^{*}}(x)-\Lambda_{0}(x)$. We have

$$
\begin{aligned}
\|z\| \leq & \left\|f\left(x^{*}\right)-f\left(x_{0}\right)-\sum_{i=1}^{M} a_{i} \nabla f\left(x_{0}+\beta_{i}\left(x-x_{0}\right)\right)\left(x^{*}-x_{0}\right)\right\| \\
+\|\left(\sum_{i=1}^{M} a_{i} \nabla f\left(x^{*}+\beta_{i}\left(x-x^{*}\right)\right)\right. & \\
& \left.-\sum_{i=1}^{M} a_{i} \nabla f\left(x_{0}+\beta_{i}\left(x-x_{0}\right)\right)\right)\left(x-x^{*}\right) \|=: D+E .
\end{aligned}
$$

With the help of (3), we have

$$
\begin{aligned}
D & =\left\|\sum_{i=1}^{M} a_{i}\left(f\left(x^{*}\right)-f\left(x_{0}\right)-\nabla f\left(x_{0}+\beta_{i}\left(x-x_{0}\right)\right)\left(x^{*}-x_{0}\right)\right)\right\| \\
& =\left\|\sum_{i=1}^{M} a_{i} \int_{0}^{1}\left(\nabla f\left(x_{0}+t\left(x^{*}-x_{0}\right)\right)-\nabla f\left(x_{0}+\beta_{i}\left(x-x_{0}\right)\right)\right) d t\left(x^{*}-x_{0}\right)\right\| .
\end{aligned}
$$

Thus,

$$
D \leq \sum_{i=1}^{M}\left|a_{i}\right|\left\|x^{*}-x_{0}\right\| \int_{0}^{1}\left\|\nabla f\left(x_{0}+t\left(x^{*}-x_{0}\right)\right)-\nabla f\left(x_{0}+\beta_{i}\left(x-x_{0}\right)\right)\right\| d t .
$$

Since $x$ and $x_{0}$ belong to $B_{\delta}\left(x^{*}\right)$, we obtain

$$
D \leq \frac{K_{1} \delta^{2}}{2} \sum_{i=1}^{M}\left|a_{i}\right|\left(1+4\left|\beta_{i}\right|\right) .
$$

In a similar way, we obtain

$$
E \leq \delta^{2} K_{1} \sum_{i=1}^{M}\left|a_{i}\right|\left|1-\beta_{i}\right| \leq \delta^{2} K_{1} \sum_{i=1}^{M}\left|a_{i}\right|\left(1+\left|\beta_{i}\right|\right) .
$$

From (15)-(17), we obtain

$$
\|z\| \leq \frac{3 K_{1} \delta^{2}}{2} \sum_{i=1}^{M}\left|a_{i}\right|\left(1+2\left|\beta_{i}\right|\right),
$$

and the inequality (9) implies $\|z\| \leq b$. 
It follows that, for all $x^{\prime}, x^{\prime \prime} \in B_{r_{0}}\left(x^{*}\right)$, by setting $F=e\left(\Psi_{0}\left(x^{\prime}\right) \cap\right.$ $\left.B_{r_{0}}\left(x^{*}\right), \Psi_{0}\left(x^{\prime \prime}\right)\right)$, we have

$$
\begin{aligned}
F \leq & e\left(\Psi_{0}\left(x^{\prime}\right) \cap B_{\delta}\left(x^{*}\right), \Psi_{0}\left(x^{\prime \prime}\right)\right) \\
\leq & L \| \sum_{i=1}^{M} a_{i} \nabla f\left(x^{*}+\beta_{i}\left(x^{\prime}-x^{*}\right)\right)\left(x^{\prime}-x^{*}\right) \\
& -\sum_{i=1}^{M} a_{i} \nabla f\left(x_{0}+\beta_{i}\left(x^{\prime}-x_{0}\right)\right)\left(x^{\prime}-x_{0}\right) \\
& -\sum_{i=1}^{M} a_{i} \nabla f\left(x^{*}+\beta_{i}\left(x^{\prime \prime}-x^{*}\right)\right)\left(x^{\prime \prime}-x^{*}\right) \\
& +\sum_{i=1}^{M} a_{i} \nabla f\left(x_{0}+\beta_{i}\left(x^{\prime \prime}-x_{0}\right)\right)\left(x^{\prime \prime}-x_{0}\right) \| .
\end{aligned}
$$

Thus

$$
\begin{aligned}
F \leq & L \| \sum_{i=1}^{M} a_{i} \nabla f\left(x^{*}+\beta_{i}\left(x^{\prime}-x^{*}\right)\right)\left(x^{\prime}-x^{\prime \prime}\right) \\
& +\sum_{i=1}^{M} a_{i} \nabla f\left(x^{*}+\beta_{i}\left(x^{\prime}-x^{*}\right)\right)\left(x^{\prime \prime}-x^{*}\right) \\
& -\sum_{i=1}^{M} a_{i} \nabla f\left(x^{*}+\beta_{i}\left(x^{\prime \prime}-x^{*}\right)\right)\left(x^{\prime \prime}-x^{*}\right) \\
& -\sum_{i=1}^{M} a_{i} \nabla f\left(x_{0}+\beta_{i}\left(x^{\prime}-x_{0}\right)\right)\left(x^{\prime}-x^{\prime \prime}\right) \\
& -\sum_{i=1}^{M} a_{i} \nabla f\left(x_{0}+\beta_{i}\left(x^{\prime}-x_{0}\right)\right)\left(x^{\prime \prime}-x_{0}\right) \\
& +\sum_{i=1}^{M} a_{i} \nabla f\left(x_{0}+\beta_{i}\left(x^{\prime \prime}-x_{0}\right)\right)\left(x^{\prime \prime}-x_{0}\right) \|,
\end{aligned}
$$

which yields

$$
\begin{aligned}
F & \leq L\left\|\left(\sum_{i=1}^{M} a_{i} \nabla f\left(x^{*}+\beta_{i}\left(x^{\prime}-x^{*}\right)\right)-\sum_{i=1}^{M} a_{i} \nabla f\left(x_{0}+\beta_{i}\left(x^{\prime}-x_{0}\right)\right)\right)\left(x^{\prime}-x^{\prime \prime}\right)\right\| \\
& +L\left\|\left(\sum_{i=1}^{M} a_{i} \nabla f\left(x^{*}+\beta_{i}\left(x^{\prime}-x^{*}\right)\right)-\sum_{i=1}^{M} a_{i} \nabla f\left(x^{*}+\beta_{i}\left(x^{\prime \prime}-x^{*}\right)\right)\right)\left(x^{\prime \prime}-x^{*}\right)\right\| \\
& +L\left\|\left(\sum_{i=1}^{M} a_{i} \nabla f\left(x_{0}+\beta_{i}\left(x^{\prime \prime}-x_{0}\right)\right)-\sum_{i=1}^{M} a_{i} \nabla f\left(x_{0}+\beta_{i}\left(x^{\prime}-x_{0}\right)\right)\right)\left(x^{\prime \prime}-x_{0}\right)\right\| .
\end{aligned}
$$


The last inequality and the choice of $\lambda$ imply

$$
F \leq L K_{1} \delta\left[\sum_{i=1}^{M}\left|a_{i}\right|\left(1+4\left|\beta_{i}\right|\right)\right]\left\|x^{\prime}-x^{\prime \prime}\right\| \leq \lambda\left\|x^{\prime}-x^{\prime \prime}\right\|,
$$

so

$$
e\left(\Psi_{0}(x) \cap B_{r_{0}}\left(x^{*}\right), \Psi_{0}\left(x^{\prime}\right)\right) \leq \lambda\left\|x^{\prime}-x^{\prime \prime}\right\|,
$$

and condition (b) in Lemma 2.1 is satisfied.

Applying this lemma we deduce the existence of a fixed point $x_{1} \in$ $B_{r_{0}}\left(x^{*}\right)$, which moreover satisfies inequality (8).

Proceeding by induction, suppose that $x_{k} \in B_{r_{k-1}}\left(x^{*}\right)$; taking $\eta_{0}=x^{*}$ and setting $r_{k}=c\left\|x_{k}-x^{*}\right\|^{2+\alpha}$, we obtain the existence of a fixed point $x_{k+1} \in B_{r_{k}}\left(x^{*}\right)$ for $\Psi_{k}$, which implies

$$
\left\|x_{k+1}-x^{*}\right\| \leq c\left\|x_{k}-x^{*}\right\|^{2+\alpha} .
$$

Continuing this process, we obtain the existence of a sequence $\left(x_{k}\right)_{k \geq 0}$ with superquadratic convergence to $x^{*}$, which completes the proof of Theorem 3.1.

Concluding remarks. Since the constant of Hölder continuity in [4] is greater than the constant of center-Hölder continuity, for some choice of the numbers $\beta_{i}$, it seems that the lower bound obtained in [4, Theorem 3.1] is greater than the lower bound obtained in the present paper.

\section{References}

[1] I. K. Argyros, An improved convergence analysis of a superquadratic method for solving generalized equations, Rev. Colombiana Mat. 40 (2006), 65-73.

[2] J.-P. Aubin, Lipschitz behaviour of solutions to convex minimization problems, Math. Oper. Res. 9 (1984), 87-111.

[3] J.-P. Aubin and H. Frankowska, Set-Valued Analysis, Birkhäuser, Boston, 1990.

[4] C. Cabuzel, A multipoint iteration formula for solving a variational inclusion in the Hölder case, submitted.

[5] C. Cabuzel and A. Pietrus, Solving a variational inclusion by a method obtained using a multipoint iteration formula, submitted.

[6] A. L. Dontchev, Local convergence of the Newton method for generalized equations, C. R. Acad. Sci. Paris Sér. I 322 (1996), 327-331.

[7] - Uniform convergence of the Newton method for Aubin continuous maps, Serdica Math. J. 22 (1996), 385-398.

[8] A. L. Dontchev and W. W. Hager, An inverse function theorem for set-valued maps, Proc. Amer. Math. Soc. 121 (1994), 481-489.

[9] A. L. Dontchev, M. Quincampoix and N. Zlateva, Aubin criterion for metric regularity, J. Convex Anal. 13 (2006), 281-297.

[10] M. H. Geoffroy, S. Hilout and A. Pietrus, Acceleration of convergence in Dontchev's iterative method for solving variational inclusions, Serdica Math. J. 29 (2003), 45-54.

[11] - - - - , Stability of a cubically convergent method for generalized equations, SetValued Anal. 14 (2006), 41-54. 
[12] M. H. Geoffroy and A. Pietrus, A superquadratic method for solving generalized equations in the Hölder case, Ricerche Math. 52 (2003), 231-240.

[13] M. H. Geoffroy, A. Pietrus and C. Jean-Alexis, A Hummel-Seebeck type method for solving variational inclusions, Optimization, to appear.

[14] A. D. Ioffe and V. M. Tikhomirov, Theory of Extremal Problems, North-Holland, Amsterdam, 1979.

[15] C. Jean-Alexis, A cubic method without second order derivative for solving variational inclusions, C. R. Acad. Bulgare Sci. 59 (2006), 1213-1218.

[16] C. Jean-Alexis and A. Pietrus, On the convergence of the Hummel-Seebeck method for variational inclusions under mild differentiability conditions, submitted.

[17] B. S. Mordukhovich, Complete characterization of openness, metric regularity and lipschitzian properties of multifunctions, Trans. Amer. Math. Soc. 340 (1993), 1-36.

[18] - Stability theory for parametric generalized equations and variational inequalities via nonsmooth analysis, ibid. 343 (1994), 609-657.

[19] A. Pietrus, Generalized equations under mild differentiability conditions, Rev. Real. Acad. Ciencias Madrid 94 (2000), 15-18.

[20] —, Does Newton's method for set-valued maps converges uniformly in mild differentiability context?, Rev. Colombiana Mat. 32 (2000), 49-56.

[21] R. T. Rockafellar, Lipschitzian properties of multifonctions, Nonlinear Anal. 9 (1984), 867-885.

[22] R. T. Rockafellar and R. J. B. Wets, Variational Analysis, Grundlehren Math. Wiss. 317, Springer, 1998.

[23] J. F. Traub, Iterative Methods for the Solution of Equations, Chelsea, 1982.

Laboratoire Analyse, Optimisation, Contrôle

Département de Mathématiques et Informatique

Université des Antilles et de la Guyane

Campus de Fouillole

F-97159 Pointe-à-Pitre, France

E-mail: catherine.zebre@univ-ag.fr apietrus@univ-ag.fr

Received on 29.8.2007;

revised version on 4.1.2008 
